\title{
High-throughput imaging of biological samples with Delmic's FAST-EM
}

Job Fermie ${ }^{1}$, Wilco Zuidema ${ }^{1}$, Radim Šejnoha ${ }^{2}$, Anouk Wolters ${ }^{3}$, Ben Giepmans ${ }^{4}$, Jacob Hoogenboom ${ }^{5}$ and Pieter Kruit ${ }^{5}$

${ }^{1}$ DELMIC B.V., United States, ${ }^{2}$ Thermo Fisher Scientific, United States, ${ }^{3}$ University Medical Center Groningen, United States, ${ }^{4}$ University Medical Center Groningen, Netherlands, ${ }^{5}$ Technical University Delft, United States

Electron microscopy (EM) is increasingly being used in large-scale biological projects, either for volume imaging or large area mapping. In both use cases, a need for increased throughput, reliability and automation of electron microscopy highlights the importance for a new approach to large-scale imaging. Here we present the advantages brought to the scientific community by FAST-EM, a multibeam scanning transmission EM system developed in collaboration between Delmic, TU Delft, Thermo Fisher Scientific and Technolution.

The imaging speed of a conventional scanning or transmission EM is a limiting factor in many large-scale workflows. At the same time, increasing the throughput of a workflow without negatively impacting image quality can be challenging. To overcome this challenge, we have developed an automated multibeam scanning EM, which combines simultaneous 64-beam electron imaging with transmission detection [1,2]. This imaging approach presents an enormous improvement for high-throughput transmission EM of biological specimens: FAST-EM increases imaging speed and reduces operator overhead, without compromising image quality.

In FAST-EM, electrons transmitted through the sample strike a scintillator plate acting as the sample substrate (Figure 1A). Local differences in sample density will transmit different amounts of electrons, and therefore produce fluctuations in light intensity [3]. Scintillation light is collected with an optical setup beneath the scintillator and recorded using a silicon photomultiplier detector array, which provides a fast method of signal generation and separation (Figure 1B). The resulting data is stored as a single, stitched image containing information from all 64 beams. The multibeam source and detectors are combined with customized stage hardware and automated software that enables extremely rapid movement and alignment of the stage with nanometer-scale positioning accuracy.

High quality images of 50-150 $\mathrm{nm}$ ultrathin sections can be acquired using FAST-EM's transmission detection path (Figure 2). Individual images are rapidly tiled to form 'megafields', comprising of many multibeam images. This workflow can be applied to a broad range of samples, such as minimally stained samples embedded in Lowicryls and also heavily stained tissues embedded in Durcupan, common in volume EM and connectomics [4]. FAST-EM's high resolution data enables clear identification of the different cell types and organelles in the pancreas (Figure 2B), while the high throughput allows for the generation of large-scale overviews at an unprecedented speed: In less than 5 minutes an area of $0.25 \times 0.25 \mathrm{~mm}(64000 \times 64000$ pixels at $4 \mathrm{~nm}$ resolution) was imaged. For the same task, a single-beam SEM, acquiring at the same dwell time, would require approximately 4 hours.

FAST-EM's multibeam electron optics combined with optical STEM detection provides a significant improvement in imaging throughput over conventional single-beam systems, without compromising on image contrast or morphological detail in biological material. FAST-EM, and high-throughput EM in general, will be instrumental in tackling current large-scale projects such as volume-EM of cells and tissues, connectomics, and large-scale 2D nanotomy projects [5]. Looking at an even broader scale, high-throughput EM opens the door for projects of unprecedented scale to be completed within reasonable time frames enabling a shift towards using EM as a fast quantitative analysis tool. 

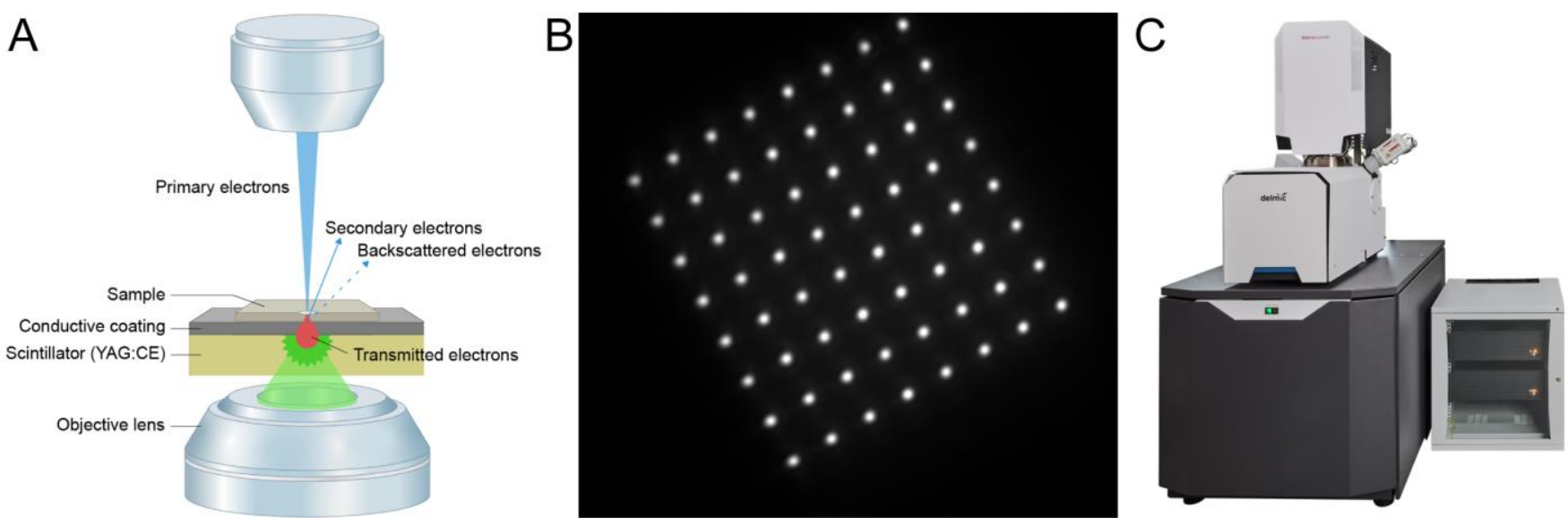

Figure 1. Multibeam imaging in FAST-EM. a. Working principles of transmission detection in multibeammode: the transmitted electrons in each of the 64 beams will hit the scintillator and produce light. The light spots produced by the beams is imaged with an objective lens mounted beneath the scintillator. b. Snapshot of the light produced by the 64 beams on the scintillator surface. Notice the $8 \times 8$ grid of the beams. c. The integrated FAST-EM system.
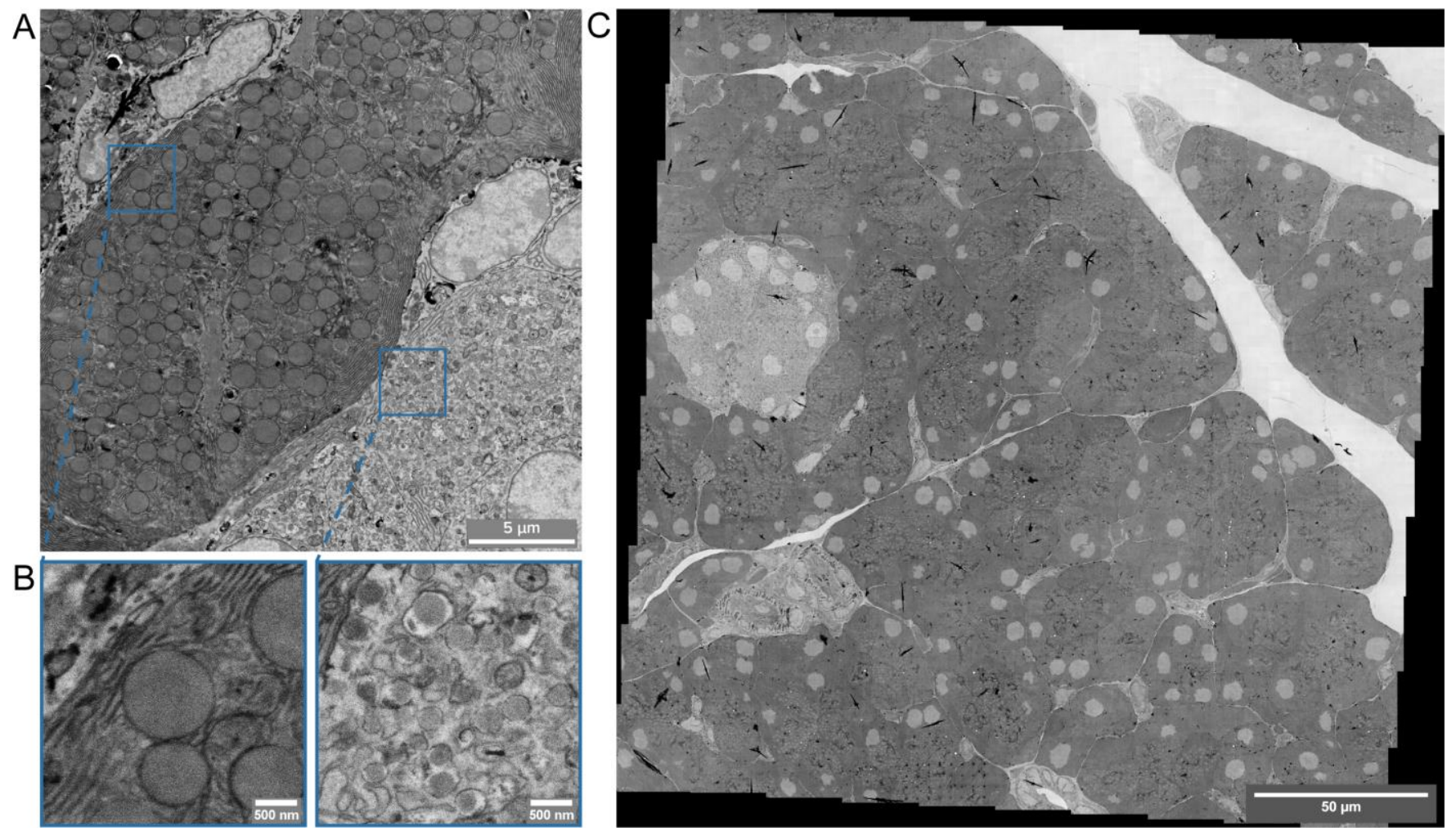

Figure 2. Multibeam STEM imaging on $0.25 \mathrm{mmx} 0.25 \mathrm{~mm}$ area of rat pancreas within 5 minutes. $80 \mathrm{~nm}$ ultrathin sections of OTO-stained, Epon-embedded pancreas tissue on scintillators was imaged at $5 \mathrm{kV}$ landing energy, $4 \mathrm{~nm}$ pixel size, $3200 \mathrm{~ns}$ dwell time. a. Typical image collected in multibeam STEM. Acquisition of a single field with the 64 -beam requires 3 seconds and covers $25.6 \times 25.6 \mu \mathrm{m}$. $\mathrm{b}$. The images collected by individual beams, which each scan $3.2 \times 3.2 \mu \mathrm{m}$. The resolution allows identification of membrane systems, organelles and macromolecular complexes (right panel). c. Megafield of the pancreas tissue, consisting of 100 multibeam images, acquired in less than 5 minutes while retaining the high image quality shown in (A) and (B). Zoomable dataset available via http://www.nanotomy.org/OA/Fermie2021M+M/index.html 


\section{References}

1] P. Kruit and W. Zuidema, "A Dedicated Multi-Beam SEM for Transmission Imaging of Thin Samples," Microsc. Microanal., vol. 25, no. S2, pp. 1034-1035, 2019.

[2] Y. Ren and P. Kruit, "Transmission electron imaging in the Delft multibeam scanning electron microscope 1," J. Vac. Sci. Technol. B, Nanotechnol. Microelectron. Mater. Process. Meas. Phenom., vol. 34 , no. 6, p. 06KF02, 2016.

[3] W. Zuidema and P. Kruit, "Transmission imaging on a scintillator in a scanning electron microscope," Ultramicroscopy, vol. 218, p. 113055, 2020.

[4] T. J. Deerinck, E. Bushong, A. Thor, and M. H. Ellisman, "NCMIR Methods for 3D EM: A New Protocol for Preparation of Biological Specimens for Serial Block-Face SEM," SBEM Protoc. v7_01_2010. Available online https//ncmir. ucsd. edu/sbem-protocol (accessed 2021-02-22)

[5] P. de Boer et al., "Large-scale electron microscopy database for human type 1 diabetes," Nat. Commun., vol. 11, no. 1, pp. 1-9, 2020. 